\title{
Collaborative Online Professional Development Design
}

\author{
Thomas Cochrane \\ Cochrane.t@unimelb.edu.au \\ Sophia Arkoudis \\ s.arkoudis@unimelb.edu.au \\ Cathleen Benevento \\ cjbe@unimelb.edu.au \\ Centre for the Study of Higher Education, University of Melbourne, Victoria, Australia.
}

Keywords: Professional Development, Research Supervision, Collaborative Design.

\begin{abstract}
:
COVID-19 has impacted not only higher education teaching practice, but also professional development (PD) and research by higher degree supervision practice, policies and systems training. Face to face workshop sessions for supervisor training and policy updates have had to pivot to online modes. This presentation overviews the process of designing an academic supervision training workshop as an online module that can be used as a design framework for other professional development and training contexts. We used a design-based research methodology (McKenney \& Reeves, 2019) encompassing three stages.
\end{abstract}

\begin{abstract}
Stage 1 Evaluation and Analysis
We formed a collaborative design team of two academics and a professional staff member and met regularly over Zoom as due to COVID-19 we were all working from home, and across countries (Australia and New Zealand). We began with an evaluation of the existing introductory supervision online module to identify key design elements and refinements that we could integrate into the new refresher module. This was followed by a collaborative definition of the scope of the new PD module that was focused upon providing a 'Refresher Course' for experienced higher degree research supervisors. The module was developed to cover seven key policy and process changes in the past 5 years as supervisors are required to refresh their training every 5 years. We decided to focus the content of the module upon authentic user-generated scenarios from highly experienced academics across the university. User reflection was designed through an optional link to contribute to an institutional Wiki page to provide tips and comments from user experiences in supervision. The analysis of users needing an informative but short time investment in the refresher module highlighted the need for collating resources that would be referenced throughout the module to be made available at the end of the module as a downloadable interactive PDF resource for users.
\end{abstract}

\section{Stage 2 Collaborative Prototype Design}

An initial storyboard module prototype based upon learning object and instructional design principles (Boyle, 2003; Cochrane, 2007; Reigeluth et al., 2016) was created using images sketched via an iPad and Apple Pencil to create a unique aesthetic and Adobe Spark was used as a rapid online deployment platform to pitch the prototype design to the stakeholders.

A series of 2-minute video vignettes formed the core content of the module, and we wanted these to be authentic and recorded by the academics themselves. We began by recording these as live Zoom meetings as we were unsure of the capacity of the users to record using their own smartphones. However, the Zoom audio was badly out of synch with the video and therefore we sent the invited video vignette producers' instructions on how to record themselves using their smartphone and share the recording via an institutional file sharing platform.

While we began with big ideas based upon the rapid prototype of how to make the new module interactive and appealing to the users there were significant design restraints imposed by the institutional Course Builder online development platform, that provides a basic Web 1.0 approach to online module design and delivery. This meant that user interaction options were limited, file size uploads were small, and display dimensions fixed. Therefore, Vimeo was used to host and embed the video vignettes in the module pages through an institutional license providing a range of privacy and customisation options. We were able to negotiate these technical design hurdles, albeit with downgraded expectations of user interaction within our new module design. 


\section{Stage 3 Evaluation and Redesign}

User feedback on the initial prototype was very positive and the user-generated video vignettes and funky iPad created graphics were highlights. Feedback also highlighted the need for additional user instructions, a more unified design across the module and implementation of accessibility features such as closed captioning for the video vignettes. This was achieved through maintaining a consistent font and paragraphing style and creating a new embedded player style format for the Vimeo video host platform. Closed captions were added by manually transcribing and timecoding the videos on Vimeo.

\section{Lessons learnt}

The presentation will highlight the lessons learnt by the design team: We learnt to keep the design simple, yet aesthetically interesting, with the main content created by recognised academic experts at the University as real user-generated vignettes. The project was an authentic collaborative design process that focused upon the scope of the learning context rather than the lack of complexity of the design and delivery platform for which we found simple work-arounds..

\section{References}

Boyle, T. (2003). Design principles for authoring dynamic, reusable learning objects. Australasian Journal of Educational Technology, 19(1). https://doi.org/10.14742/ajet.1690

Cochrane, T. (2007, Available online 20 September 2006). Developing interactive multimedia Learning Objects using QuickTime [Internet]. Computers in Human Behavior, 23(6), 2596-2640.

https://doi.org/10.1016/j.chb.2006.08.007

McKenney, S., \& Reeves, T. (2019). Conducting educational design research (2nd ed.). Routledge. https://doi.org/10.4324/9781315105642

Reigeluth, C. M., Beatty, B. J., \& Myers, R. D. (2016). Instructional-design theories and models, Volume IV: The learner-centered paradigm of education. Routledge. https://www.routledge.com/Instructional-DesignTheories-and-Models-Volume-IV-The-Learner-Centered/Reigeluth-Beatty-Myers/p/book/9781138012936 